The Hospice UK audit will be repeated in Spring 2018 in order to monitor progress.

\section{P-157 IMPROVING THE PATIENT EXPERIENCE OF WOUND/ PRESSURE ULCER CARE IN A HOSPICE SETTING}

Louise Wonham, Lynn Cornish. St Margaret's Hospice, Taunton, UK

\subsection{6/bmispcare-2017-hospice.182}

Background Holistic patient- centred care is an essential part of palliative care, and wound and pressure ulcer management is no exception. Previously, patients were kept waiting for a competent nurse to assess and treat their wound/pressure ulcer. This practice resulted in delayed healing and exacerbation of disease symptoms. Relatives' visits were interrupted, which reduced the valuable time spent with loved ones. Nurses lacked knowledge and confidence to assess and treat complex wounds/pressure ulcers, and felt that their practice was not evidence based and current.

Aim To improve the patient and relative experience, by introducing a training programme which would ensure that all staff were competent and confident to implement evidence based and current best practice.

Methods We reviewed the old system of provision of wound/ pressure ulcer prevention and management, and discussed how it could be improved. We designed a new strategy of wound/ pressure ulcer provision, which included an education and ward- based training programme for all staff, which we felt would resolve those issues which staff felt did not facilitate the best patient and relative experience. The programme consisted of a full day mandatory training educational day for all registered nurses and healthcare assistants, mandatory ward based competencies, and annual updates. The results of this strategy have been evaluated through a research study which has formed the dissertation of one of the authors MSc in wound healing and tissue repair.

Results

- Staff of all grades are now able to provide a gold standard wound/pressure ulcer service

- Patient experience has improved

- Relatives' experience has improved

- Documentation has improved

- Efficiency on the ward has improved.

Conclusions We believe that the implementation of this new strategy has improved the patient and relative experience, and improved service provision. We also believe that this strategy could be tested and duplicated in other settings.

\section{P-158 COLLABORATIVE WORKING WITH HEALTH AND SOCIAL CARE AGENCIES TO REDUCE AVOIDABLE PRESSURE ULCERS}

Sally Newman, Jane Pickard. LOROS Hospice, Leicester, UK

\subsection{6/bmispcare-2017-hospice. 183}

Background Patients being cared for palliatively are at increased risk of developing pressure ulcers due to multiple intrinsic and extrinsic risk factors, therefore a robust process for preventing pressure ulcers is a crucial aspect of providing safe patient care to this patient group.
Aim To work collaboratively with local partners in health and social care and commissioners to increase the profile of pressure ulcer prevention to health and social care staff, patients, carers and the general public.

Method A strategic group including Clinical Leaders and Tissue Viability Nurses from each sector met monthly to discuss how to empower health and social care staff, patients, carers and the general public to reduce the risk of pressure ulcer development. This resulted in the development and implementation of the following;

The development of a standardised education package regarding identifying, preventing and treating pressure ulcers which was delivered to social and healthcare staff throughout Leicestershire. This package was supplied to all local nursing agencies and delivered face to face to the agency used by the Unit.

Monthly figures for each organisation were shared at each meeting and geographical areas of concern noted, with action plans made to address these.

A leaflet was developed to alert patients and carers to early signs of pressure damage to encourage them to seek help. This was used to educate patients and carers throughout the organisations.

Results Positive working relationships have been developed between the sectors. This has enhanced care for patients as they now receive more consistent care between the settings as the staff are receiving the same training and share appropriate information. For LOROS the incidence of hospice acquired pressure ulcers has reduced by $19 \%$ in comparison with the last financial year and sharing good practice has facilitated the development of robust assessment, implementation and documentation of pressure ulcer prevention, identification and treatment strategies.

\section{P-159 USING APPRECIATIVE INQUIRY TO EXPLORE AND FACILITATE CONVERSATIONS OF ACQUIRED GRADE 2 PRESSURE ULCERS}

Melanie Legg, Dee Sissons, Anne Cleary. Marie Curie, London, UK

\subsection{6/bmjspcare-2017-hospice. 184}

Background Appreciative Inquiry (AI) is a way of looking at organisational change which focuses on identifying and doing more of what is already working, rather than looking for problems and trying to fix them. AI may be used to facilitate workforce engagement, and promote organisational learning and positive organisational change in the health care context (Trajkovski et al., 2013). Pressure ulcers were seen as a way of exploring the use of $\mathrm{AI}$ and its potential impact in culture change.

Aims

- To provide a safe space and facilitate engagement with teams when exploring case studies

- To explore learning from the case studies and how this was being shared/disseminated

- To develop a culture of honesty, openness and trust ensuring Duty of Candour.

Methods Grade 2 pressure ulcers were flagged on our reporting systems and the Director of Nursing was notified. The team was then extended an invitation to present this case study to the Director and her team to explore this scenario and any learning that has come from it. 
Results Currently a work in progress and we are in the process of evaluating this work.

Conclusion A culture of engagement is more likely to predict performance than any other variables including competence (Alimo-Metcalfe \& Bradley, 2010). Through discussion and exploration we are able to expand upon learning and explore decision making. Disseminated learning allows for more openness and transparency amongst the team. There is a shift from the perception of a 'blame culture' to one of collaboration, inclusiveness and participation. Including staff who delivered the front-line care in these scenarios and discussions further enriches the process.

\section{P-160 THE BOTTOM LINE - NO PRESSURE!}

Anne Marie Jones. Garden House Hospice Care, Letchworth Garden City, UK

\subsection{6/bmispcare-2017-hospice.185}

Managing pressure injuries within palliative care patients has been shown to be challenging due to, decreased nutritional intake, reduced mobility and increasing frailty resulting from co-morbidities. Included in this would be patients taking steroids and those with complications from treatment which would compromise their skin integrity. Due to high numbers of patients being admitted to the inpatient unit with pressure injuries it was felt that reducing further deterioration of their pressure areas was paramount.

Aim The Tissue Viability Link nurse role is to initiate and incorporate creative ways to educate staff about the importance of preventing deterioration of pressure injuries in our patient group and keep staff up- to- date with the latest initiatives.

Method - A tissue viability focus group was developed to look at areas to improve the management of pressure injuries within the hospice

- A new policy has been devised incorporating the SSKIN algorithm and NICE guidelines

- Training sessions developed and delivered at nursing handovers and at away days

- A laminated pocket guide produced to enable staff to grade pressure injuries. An enlarged version of this was made for all the patient folders

- A patient and carer information leaflet produced about managing pressure injuries

- Heel mirrors purchased and informative sign produced so staff are reminded to use them

- Guidelines on type of wound dressings produced to streamline which dressings would be used

- 'The Bottom line' newsletter published bi-monthly covering relevant information for staff, interesting facts, 'Don't forget' section, 'News in Brief' section to keep staff updated

- SSKIN charts which are helping to prompt staff with repositioning our patients.

Results Mini audit and Hospice UK audits carried out have shown that the staff's knowledge and ability to recognise and put measures in place to minimise pressure injuries and complete necessary documentation has greatly improved.

Recommendations To continue to publish newsletter for staff and find innovative ways to raise staff awareness about ways to implement preventative measures. To continue to carry out Hospice UK audits within the allocated timeline.

\section{P-161 IMPROVING THE CONTENT AND TIMELINESS OF DISCHARGE LETTERS FROM A HOSPICE INPATIENT UNIT}

Joanne Rimmer, Aruna Hodgson. Wigan and Leigh Hospice, Greater Manchester, UK

\subsection{6/bmjspcare-2017-hospice. 186}

Background When a patient is discharged from an inpatient setting back into the community, essential information about their condition and its treatment must be communicated accurately and efficiently via a comprehensive discharge letter to the relevant health care professionals.

Aims To audit the content and timeliness of discharge letters from a hospice inpatient unit and rectify deficiencies identified by agreeing and implementing effective interventions.

To re-audit the impact of changes made, thereby completing the audit cycle.

Methods An initial audit identified that the standards for hospice discharge letters were not being met in several areas. Following this, four main strategies were implemented to achieve improvements:

1. Introduction of a discharge letter proforma

2. Enhanced communication systems between the medical team and clinical secretaries

3. Discharge letters faxed, rather than posted to ensure timeliness of delivery

4. Ongoing monitoring of when letters were being sent, with quarterly reports presented to the medical team.

One year following introduction of these changes, a retrospective re-audit was undertaken of twenty-five consecutive patients discharged from a hospice inpatient unit.

Results All areas of deficiencies previously identified were improved upon in the re-audit. The discharge letter proforma succeeded in standardising the format and content of letters, ensuring key positive and negative information was recorded. The systems put in place to ensure timeliness of letter-sending resulted in $96 \%$ being sent out within one working day, (versus $45 \%$ previously). These improvements have enhanced communication between the hospice, hospital and community.

Conclusions Further alterations to hospice discharge letters and ongoing monitoring are required to maintain and optimise standards. The audit work undertaken has demonstrated that effective clinical changes can be achieved through collaboration with colleagues to agree achievable action plans, and by ongoing good team working to attain high standards and additonal improvements in practice.

\section{P-162 ENHANCING DISCHARGE PROCESSES}

Tracy Parkinson, Janet McGeown. St Catherine's Hospice, Preston, UK

\subsection{6/bmjspcare-2017-hospice. 187}

Background Review of discharge processes was identified as a workstream within an overall in-patient unit project modernising ways of working to meet current service demands. Staff feedback showed increasing anxiety relating to completion of the discharge process and documentation, in particular relating to continuing health care funding. Anecdotally it was also felt that patient and family expectations of ongoing hospice care were a barrier to timely discharge planning.

Aims

1. Increase in number of patient discharges

2. Reduction in length of stay 